\title{
High-resolution visible and infrared imaging for large paintings: a case study of Israel in Egypt by Poynter
}

C. Willard, A. Gibson, N. Wade

C. Willard, A. Gibson, N. Wade, "High-resolution visible and infrared imaging for large paintings: a case study of Israel in Egypt by Poynter," Proc. SPIE 11058, Optics for Arts, Architecture, and Archaeology VII, 110581F (12 July 2019); doi: $10.1117 / 12.2525714$

SPIE. Event: SPIE Optical Metrology, 2019, Munich, Germany 


\title{
High-resolution visible and infrared imaging for large paintings: a case study on Israel in Egypt by Poynter
}

\author{
C. Willard*a,b, A. Gibson ${ }^{\mathrm{a}}$, N. Wade ${ }^{\mathrm{c}}$ \\ ${ }^{a}$ Dept. of Medical Physics and Biomedical Engineering, University College London, UK; \\ ${ }^{\mathrm{b}}$ Institute for Sustainable Heritage, University College London, UK; \\ ${ }^{\mathrm{c}}$ Guildhall Art Gallery, City of London, UK.
}

\begin{abstract}
Israel in Egypt is an oil on canvas painting by Sir Edward Poynter. In 1868 the painting was depicted in the Illustrated London News in which there are reports of changes made to the composition of the painting after its first exhibition. Visible and infrared imaging techniques have been used to determine whether additions to the initial composition can be identified from underdrawings. The painting measures $137 \mathrm{~cm}$ x $317.5 \mathrm{~cm}$ and was not able to be relocated for the study, therefore portable imaging equipment was used throughout. A Canon 700D DSLR camera was modified to allow nearinfrared imaging when combined with a set of longpass filters at $720 \mathrm{~nm}, 850 \mathrm{~nm}$ and $950 \mathrm{~nm}$. An Osiris infrared reflectography camera was also used to look further into the infrared with a sensitivity range of $900 \mathrm{~nm}-1700 \mathrm{~nm}$. To obtain high-resolution images with the modified DSLR, a $100 \mathrm{~mm}$ lens was used from a distance of 6 metres. In both visible and near-infrared, eight images were taken across the surface of the painting and these images were combined into high-resolution visible and near-infrared panoramas. Images from the Osiris infrared camera were processed in Matlab to create a mosaic from the overview image with high-resolution regions of interest. All processed images were registered in Matlab along with the woodcut engraving of the painting shown in the Illustrated London News. An interactive web-browser viewer was created to enable display and comparison of the registered high-resolution images, allowing users to explore and zoom in to specific areas of interest across the four high-resolution images simultaneously. Conservators and art historians can utilise the resulting images combined with the image viewer to analyse the painting and potentially develop a new interpretation of the composition.
\end{abstract}

Keywords: Imaging, high-resolution, infrared, spectral, image stitching, image registration

\section{INTRODUCTION}

Israel in Egypt by Sir Edward Poynter was first displayed at the Royal Academy summer exhibition in 1867. The painting is particularly interesting as it was depicted in the Illustrated London News (ILN) in January 1868 which reports on additions made to the painting after its initial exhibition ${ }^{1}$. There has been no previous analytical imaging of the painting and this study investigates the use of infrared imaging techniques at different wavelengths and resolutions to analyse the painting.

*Charles.Willard.17@ucl.ac.uk

Optics for Arts, Architecture, and Archaeology VII, edited by Haida Liang,

Roger Groves, Piotr Targowski, Proc. of SPIE Vol. 11058, 110581F

(c) 2019 SPIE · CCC code: 0277-786X/19/\$21 - doi: 10.1117/12.2525714

Proc. of SPIE Vol. 11058 110581F-1 


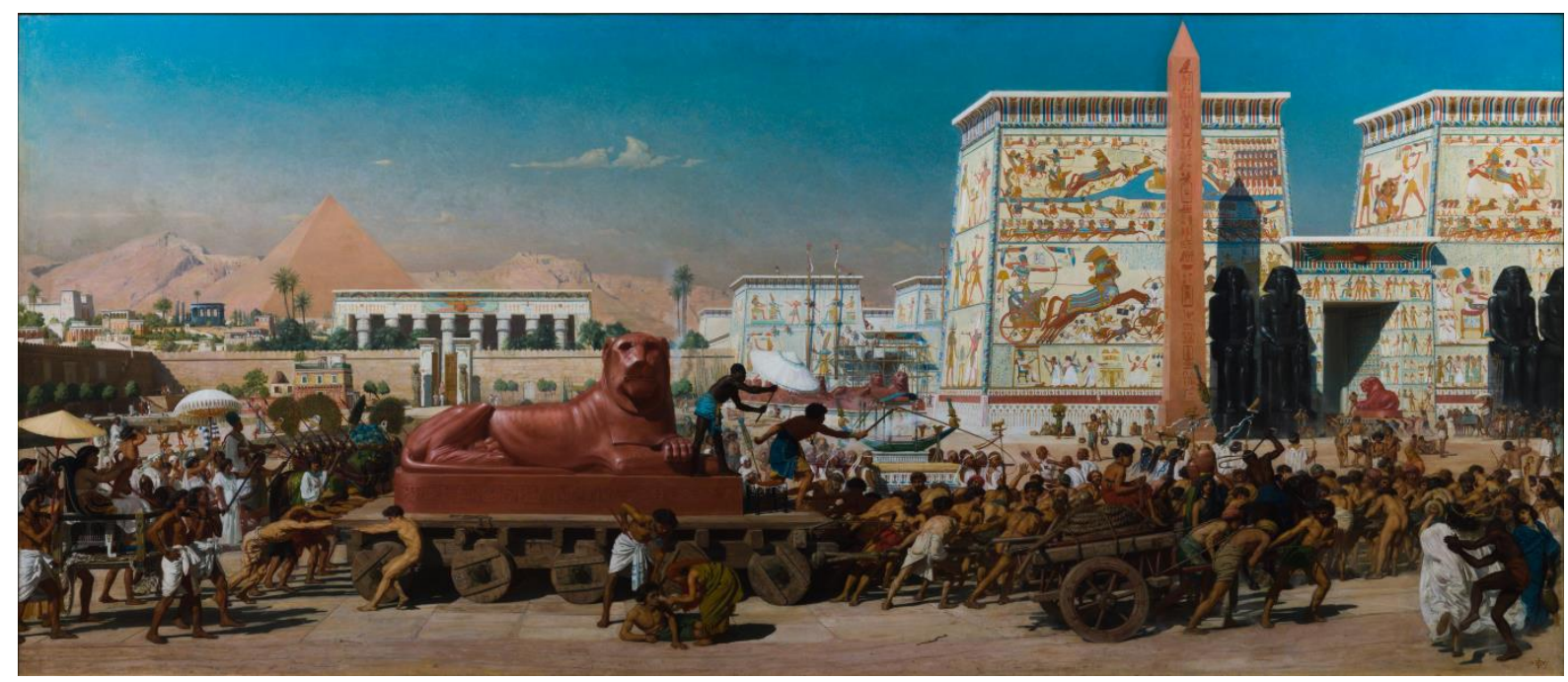

Figure 1 - 'Israel in Egypt' oil on canvas painting by Sir Edward Poynter, 1867, measuring $317.5 \mathrm{~cm}$ x $137 \mathrm{~cm} .{ }^{2}$

The Illustrated London News featured a letterpress woodcut-engraving of the painting and reports on additions made after the engraving was cut. Particular areas of interest are additions to pulling figures towards the front, continuations to the royal procession as well as priests carrying scared emblems and women playing on harps.

It is likely that the engraving would have been made using a photograph of the painting taken whilst it was on display at the Royal Academy. With the advent of the collodion process in 1851 it was possible to print the photographic image directly onto the close-grained box wood block which would then be carved into using a small sharp tool called a graver ${ }^{3}$.

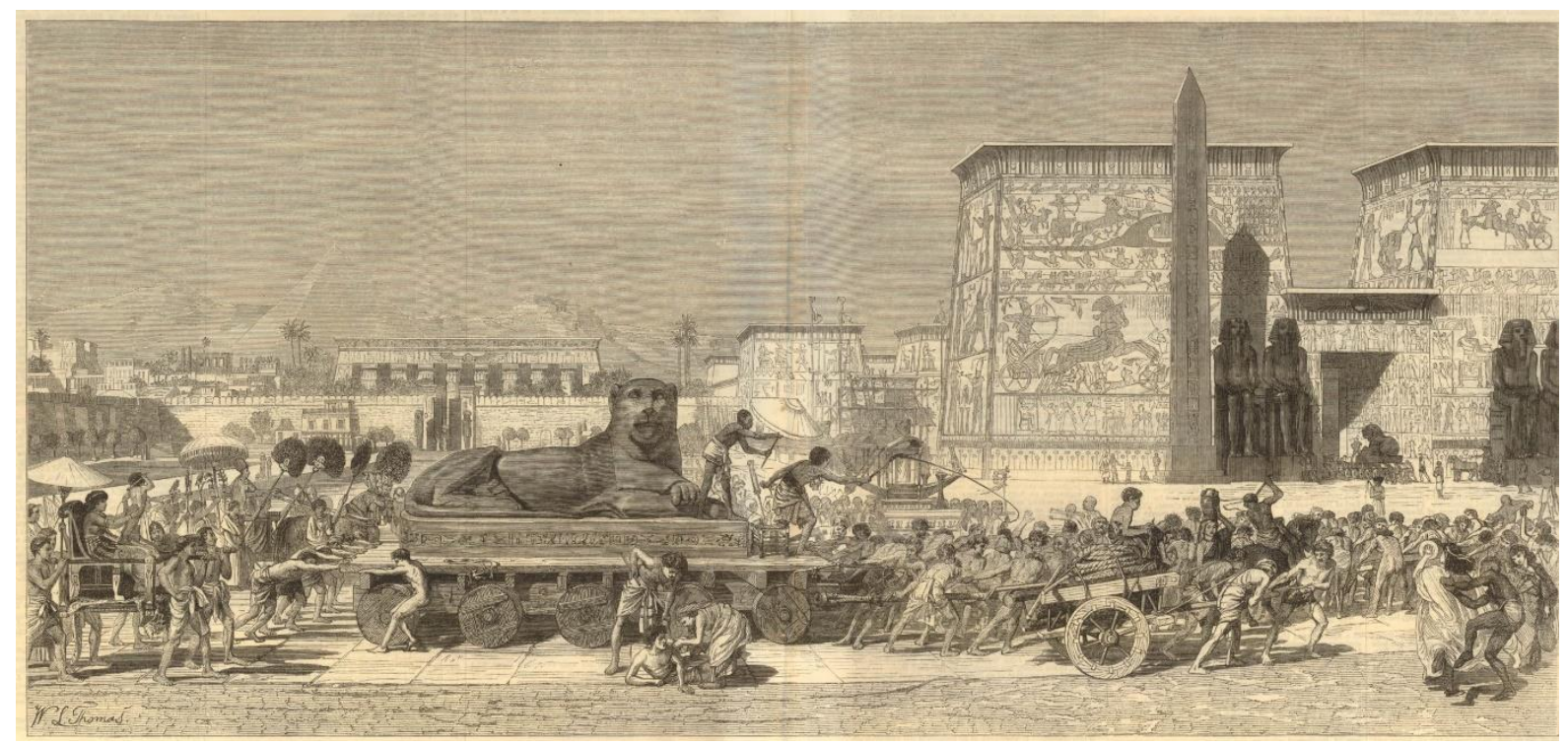

Figure 2 - 'Israel in Egypt' as shown in the Illustrated London News ${ }^{4}$. () Trustees of British Museum

Infrared imaging is well established and is often used to reveal the underdrawing of a painting ${ }^{5,6,7}$. In this study we imaged the painting in visible, near infrared (NIR) and short wave infrared (SWIR) at varying resolutions. The resulting image set varies in resolution and wavelength range. 
This study investigates multimodal and multiscale registration addressing the following research questions:

- Can images from different modalities and varying resolutions be accurately registered?

- How can the resulting high-resolution images be usefully displayed for interpretation?

- Can changes to the painting composition be determined from the infrared images?

\section{METHOD}

\subsection{DSLR acquisition}

A Canon 700D DSLR camera (18 MP) was modified by removing the internal infrared blocking filter and replacing it with clear glass, this allows the camera to capture NIR images when appropriate filters are used in front of the lens. To capture visible light images, an original white balance (OWB) filter was used to restore the original functionality of the camera by replacing the infrared blocking filter in a more accessible position behind the lens.

For this study, three infrared longpass filters were used; $720 \mathrm{~nm}, 850 \mathrm{~nm}, 950 \mathrm{~nm}$. The spectral transmission of these filters was tested in a lab environment using a calibrated OceanOptics 2200 spectrometer. The spectrometer was aimed towards a Spectralon tile, which has a diffuse reflectance $>95 \%$ across wavelengths $250 \mathrm{~nm}$ to $2500 \mathrm{~nm}$. The Spectralon tile was illuminated by a halogen lamp using an Osram Halogen Superstar $400 \mathrm{~W}$ bulb. A reference spectrum was collected before each filter was placed in the path in front of the spectrometer. The transmittance was calculated as the ratio of intensity measured through the filter against the reference intensity with no filter, accounting for dark noise. Results showing the transmittance for each filter are shown in Figure 3.

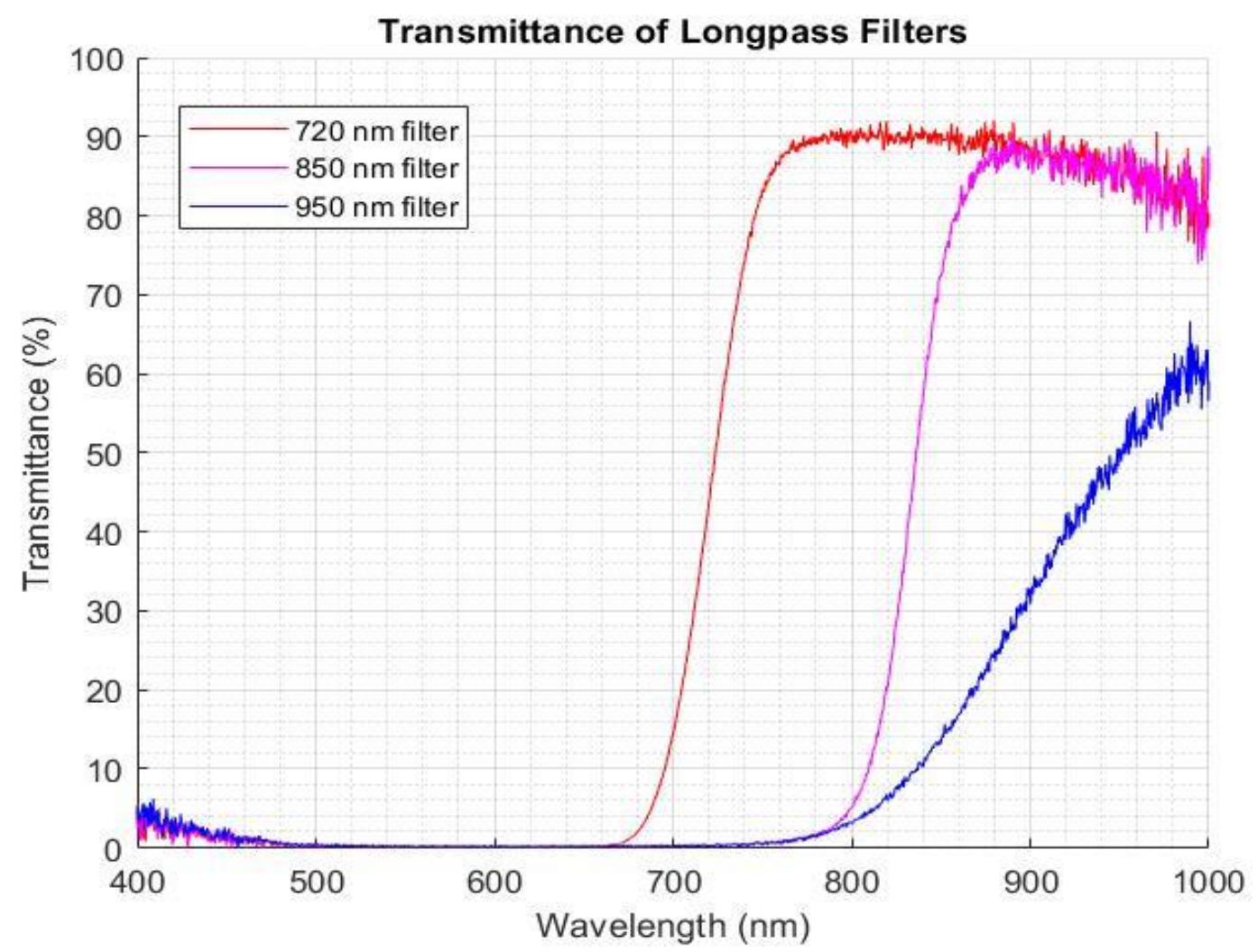

Figure 3 - Showing the transmittance of the different longpass filters used with the modified DSLR. 
Overview images were taken using a Canon EF 50mm f/1.8 STM lens to capture the entire painting in one image at $7.5 \mathrm{~m}$ distance. Images were taken for each filter setup - Visible (RGB), IR $720 \mathrm{~nm}$, IR $850 \mathrm{~nm}$ and IR $950 \mathrm{~nm}$. The infrared images are taken using the same camera settings to allow for direct radiometric comparisons in processing and analysis. For each image, a colour checker was placed in front of the painting to correct for illumination and camera response, the illumination was not adjusted throughout the DSLR imaging. The overview images were captured at a resolution of 0.65 $\mathrm{mm} /$ pixel.

To capture a high-resolution panoramic image of the painting a Canon EF $100 \mathrm{~mm} \mathrm{f} / 2.8 \mathrm{~L}$ Macro IS USM lens was used from $6 \mathrm{~m}$ distance and 8 images were taken across the surface forming a grid of two rows and four columns. Each image tile was captured in visible light, with the OWB filter behind the lens, and again with the $950 \mathrm{~nm}$ infrared longpass filter mounted to the lens with the OWB filter removed. Images of a colour checker placed in the centre of each image tile were also taken for calibration. The panoramic images were captured at a resolution of $0.25 \mathrm{~mm} / \mathrm{pixel}$.

\subsection{Osiris acquisition}

Osiris is an infrared reflectography camera manufactured by Opus Instruments using an InGaAs sensor which is sensitive to wavelengths from $900 \mathrm{~nm}$ to $1700 \mathrm{~nm}^{8}$. The Osiris system uses a scanning sensor which moves behind the $150 \mathrm{~mm}$ lens to capture a $16 \mathrm{MP}$ image over 10 minutes.

To capture the entire painting in one image, the camera was positioned $4 \mathrm{~m}$ from the painting. One scan of the total scan area was completed resulting in a 16MP image containing the entire painting in one image. The lights were in the same position as for the DSLR imaging and the 50HZ Osiris pre-set profile was used to capture the images with f5.6 aperture.

Due to the lack of interchangeable lenses allowing greater magnification, the camera was moved closer to the painting to capture regions of interest in greater detail. As the camera was moved close to the painting $(\sim 70 \mathrm{~cm})$, the lights were also moved to increase intensity locally around the region of interest, whilst minimising specular reflections. The Osiris overview image was taken at a resolution of $0.8 \mathrm{~mm} / \mathrm{pixel}$, and regions of interest were acquired at $0.3 \mathrm{~mm} / \mathrm{pixel}$ and $0.05 \mathrm{~mm} /$ pixel.

\subsection{Processing}

The colour correction pipeline relies on free and open source software; Adobe DNG Converter', ColorChecker Passport v1.1.1 ${ }^{10}$ and RawTherapee v5.411. Raw images in .CR2 format were converted into Adobe DNG format, these files were then processed in ColorChecker Passport software where the colour checker was automatically detected to generate a colour profile. RawTherapee v5.4 was used to apply this colour profile to the raw .CR2 files, the output was saved as a 16-bit uncompressed TIFF file.

The visible overview image was corrected using the colour correction pipeline described above. All overview images were then registered in Matlab using an evolutionary optimiser and Mattes mutual information metric ${ }^{\mathbf{1 2}}$.

Each colour band of the image represents the transmittance through two filters; the infrared longpass filter and the colour bandpass filter from the Bayer pattern on the camera sensor. These filters may have different properties in the infrared region, resulting in different properties for each colour channel. 
A histogram of the overview image with $950 \mathrm{~nm}$ longpass filter is shown in Figure 4, from this histogram we can see that the red and blue channels are more sensitive to the infrared light. As the green channel is less sensitive, the signal to noise ratio of this channel is lower than red and blue channels. The green channel was ignored and an average of the intensity recorded in the red and blue channels has been used to generate a greyscale image.

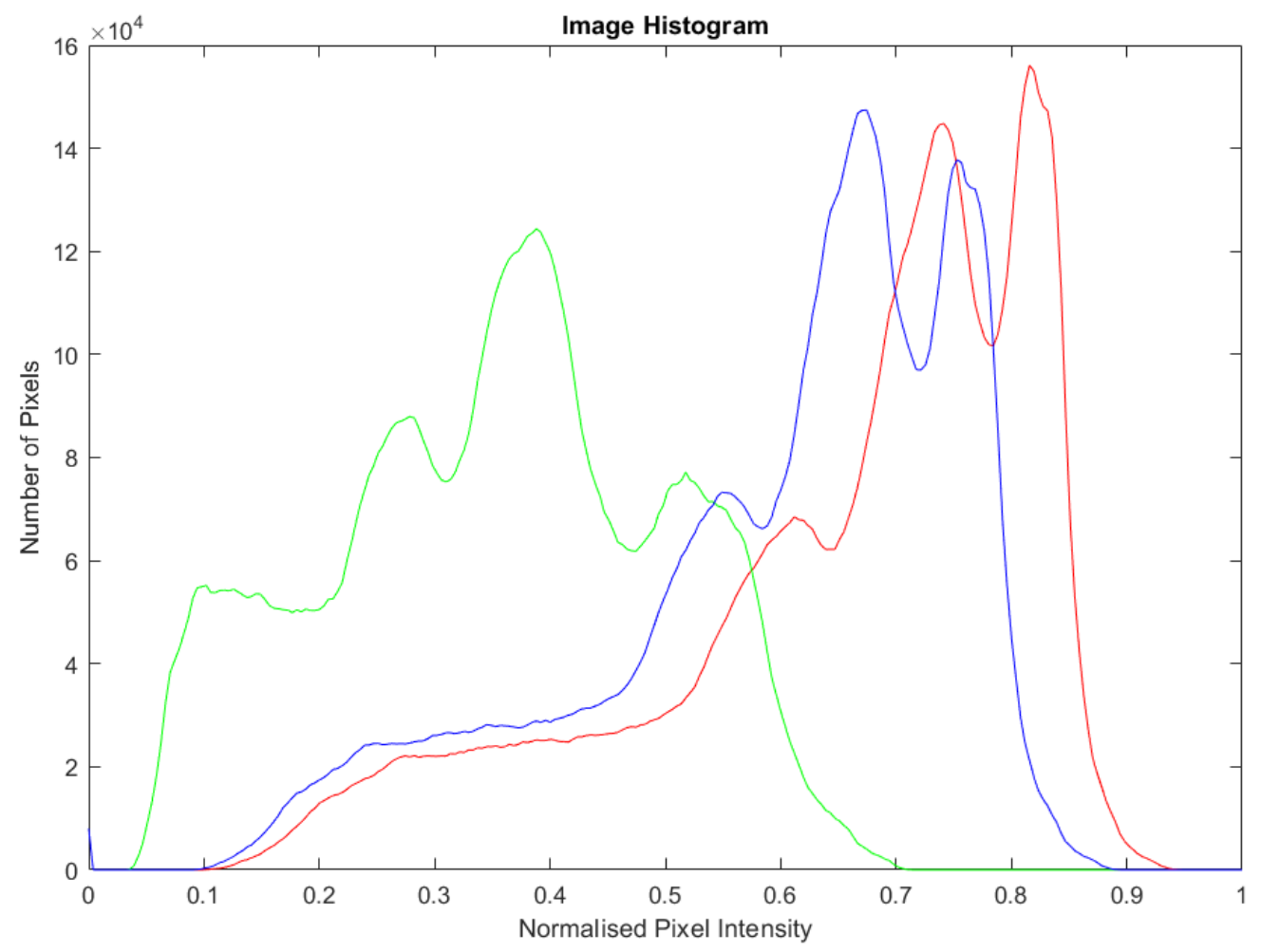

Figure 4 - Image histogram for the overview image with $950 \mathrm{~nm}$ longpass filter, showing difference between each colour channel.

To generate a high-resolution visible image, each image tile was colour corrected using the pipeline described above. Each corrected tile was then processed in PTGui Pro v11.9 ${ }^{13}$ which was able to stitch the images together into a seamless panorama. The overview image was also used as an anchor image to reduce distortion, though the overview image was not included in the resulting panorama.

This process was repeated for the infrared images taken through a $950 \mathrm{~nm}$ longpass filter mounted on front of the $100 \mathrm{~mm}$ lens. The infrared images were also processed using the colour profiles created from the visible imaging. This was done as the ColorChecker software was not able to produce a colour profile for images taken through infrared longpass filters. As the illumination was the same for both visible and infrared images, the colour profiles were used to correct for broad intensity variation across the image tiles.

As the Osiris camera was moved to capture high-resolution images at regions of interest, the PTGui software could not be used to generate a panorama because this requires the camera to pan and tilt from the same position. To generate a mosaic image from the Osiris image set, the images were processed in Matlab using SURF features to estimate the projective transforms ${ }^{14}$. This allows the Osiris images to be displayed as one high-resolution mosaic with regions of interest which can be shown at high-resolution. 
The panoramic and mosaic images were registered together in Matlab using Mattes mutual information metric, and evolutionary optimizer to estimate affine transforms ${ }^{\mathbf{1 2}}$. The image taken from the DSLR with $950 \mathrm{~nm}$ longpass filter was registered to the visible image, then the Osiris image was registered to the registered $950 \mathrm{~nm}$ longpass image. The image from the ILN was then registered to the registered Osiris image. This was done as there is likely to be some overlap in spectral sensitivity between the $950 \mathrm{~nm}$ longpass image and the Osiris image, which should result in better registrations. This was repeated for the high-resolution panoramic and mosaic images.

\section{RESULTS \& DISCUSSION}

The registered high-resolution images are displayed in an interactive web-browser viewer developed using Javascript to allow the synchronised and simultaneous investigation across the high-resolution images of the painting. Table 1 shows the layout of the interactive viewer and the images shown in each part of the display.

Table 1 - Layout of the interactive viewer.

\begin{tabular}{|c|c|}
\hline Visible panorama & $\begin{array}{c}\text { Illustrated London News } \\
\text { (C) Trustees of British Museum }\end{array}$ \\
\hline Near infrared $950 \mathrm{~nm}$ panorama & Osiris mosaic \\
\hline
\end{tabular}

The images have been effective in revealing information about the artist's technique and identifying changes made to the composition. From the infrared images it becomes apparent that Poynter has used ruled construction lines particularly in the placement of the built structures. The precise of drawing of the pyramid has been assisted by a ruled vertical line running though the centre of the pyramid and ruled construction lines forming a right angle to the top and right of the pyramid. A combination of ruled and freehand lines have been used for the outer edges as shown in Figure 5. Diagonal lines have been used to construct the stone pavement in the foreground and many of these lines have been followed in the painting stage.

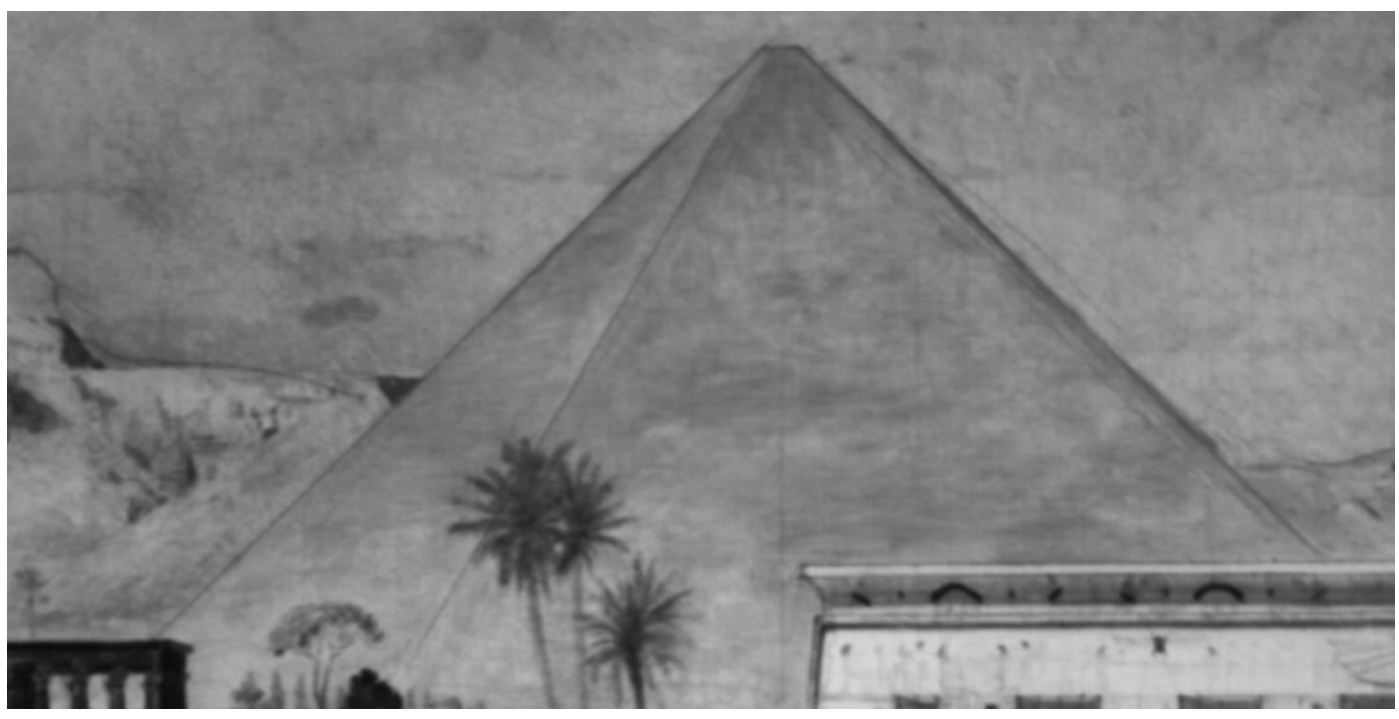

Figure 5 - An Osiris image of the Pyramid, with a vertical construction line visible through the centre of the pyramid. 
It is possible to see planned and unplanned components of the composition. The main outlines of each structure have been drawn with a ruler then detailed forms have been drawn on top, for example the two stone pillars flanking the gate go over the long baseline drawn for the perimeter wall. In the temple each column has been carefully measured out with a ruled vertical central line, however Poynter has not included any underdrawing at all to indicate the foliage in front of the columns. It is clear that there is no underdrawing for the foliage because the pigments used to paint the foliage are transparent in the Osiris image allowing us to see the completed forms of the columns with the deep shadow painted at either side and no sketched outline or indication of foliage. This suggests that the foliage was freely painted at a later stage over the top of the completed columns and shadows as shown in Figure 6.

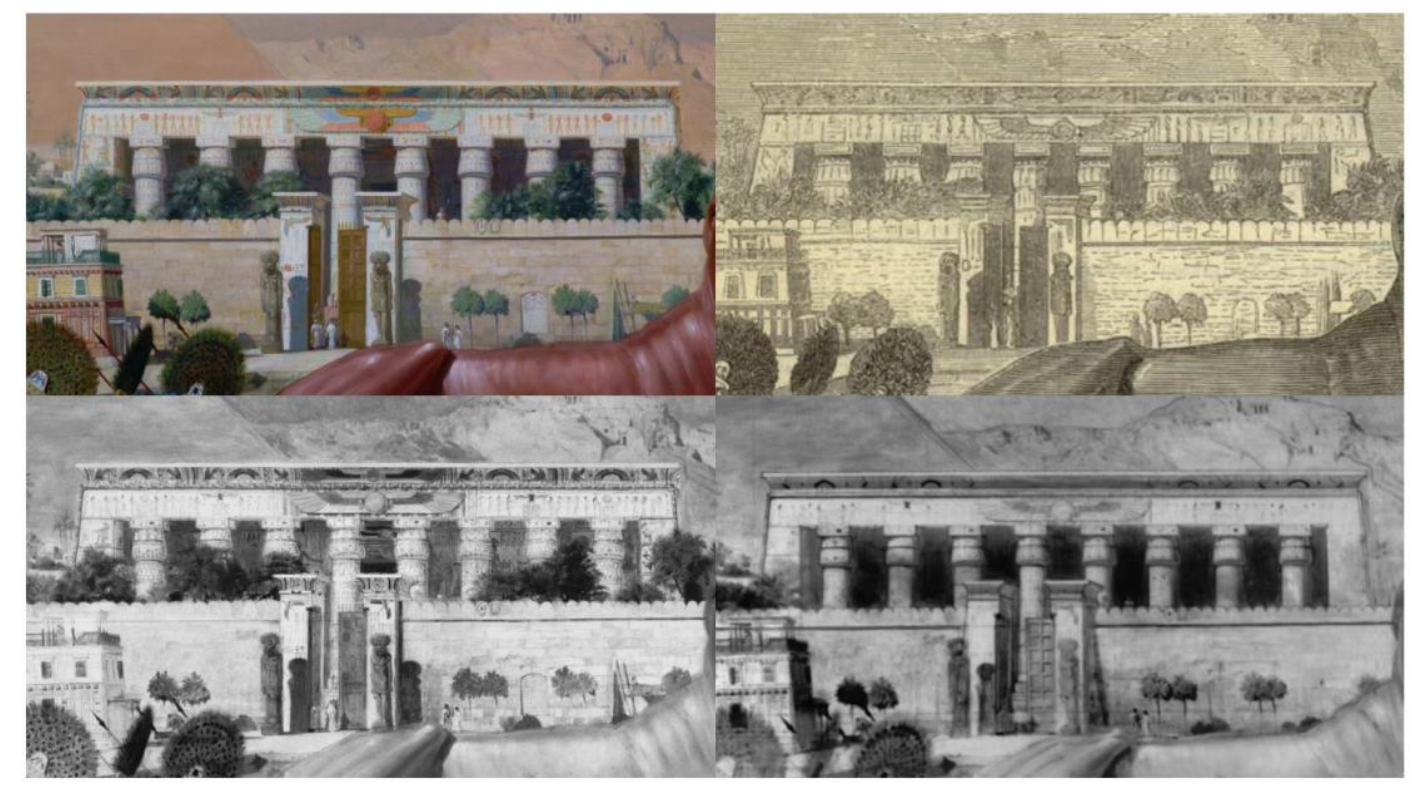

Figure 6 - Showing the temple in the interactive viewer, the foliage is visible in all images except the Osiris image (bottom right).

Poynter made numerous changes or pentimenti to his composition and some of these can be observed in the infrared images looking at the underdrawing and painting stages. It is interesting to note that these changes were made to both significant and seemingly insignificant elements of the composition alike. Arguably the most significant alteration to the underdrawing concerns the placement of the obelisk. 
Poynter moved the obelisk about $2 \mathrm{~cm}$ to the right after the sky had been painted, suggesting that he was not happy with his initial composition. This can be followed through a series of ruled lines in the area and the use of a blue paint comprised of a different pigment to the original sky to fill in the reserve to the left of the top of the obelisk as shown in Figure 7.

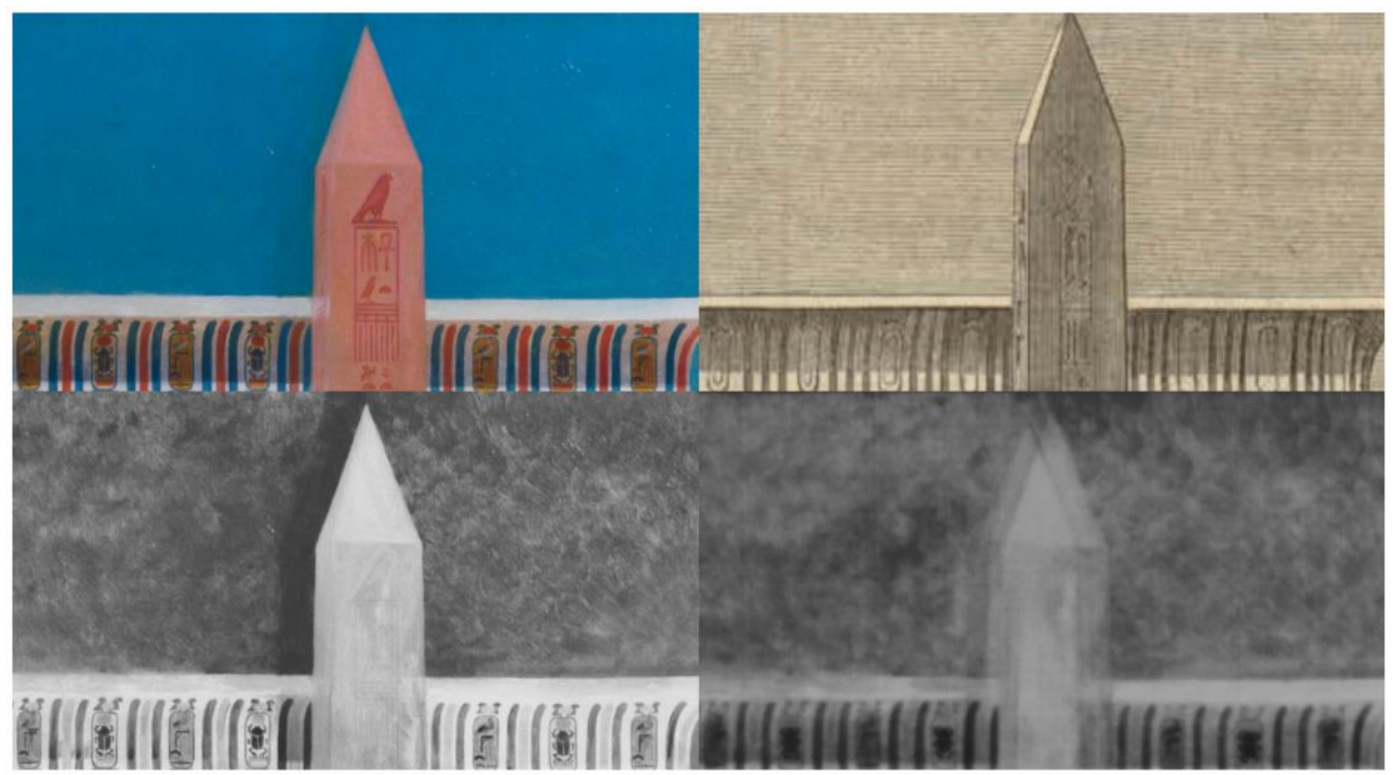

Figure 7 - Showing the tip of the obelisk in the interactive viewer - it is possible to see changes made to its position in the Osiris image (bottom right).

The top of the pyramid also appears to have been altered, in the initial drawing it had a truncated top and only received its pinnacle in the painting stage as seen in Figure 8. The great pyramid at Giza has a truncated pinnacle as its pointed capstone is missing. It may be that Poynter originally wanted to faithfully depict the great pyramid of Giza, albeit relocated to a fictitious setting without its two companion pyramids. However, after painting the sky, the pinnacle was added.

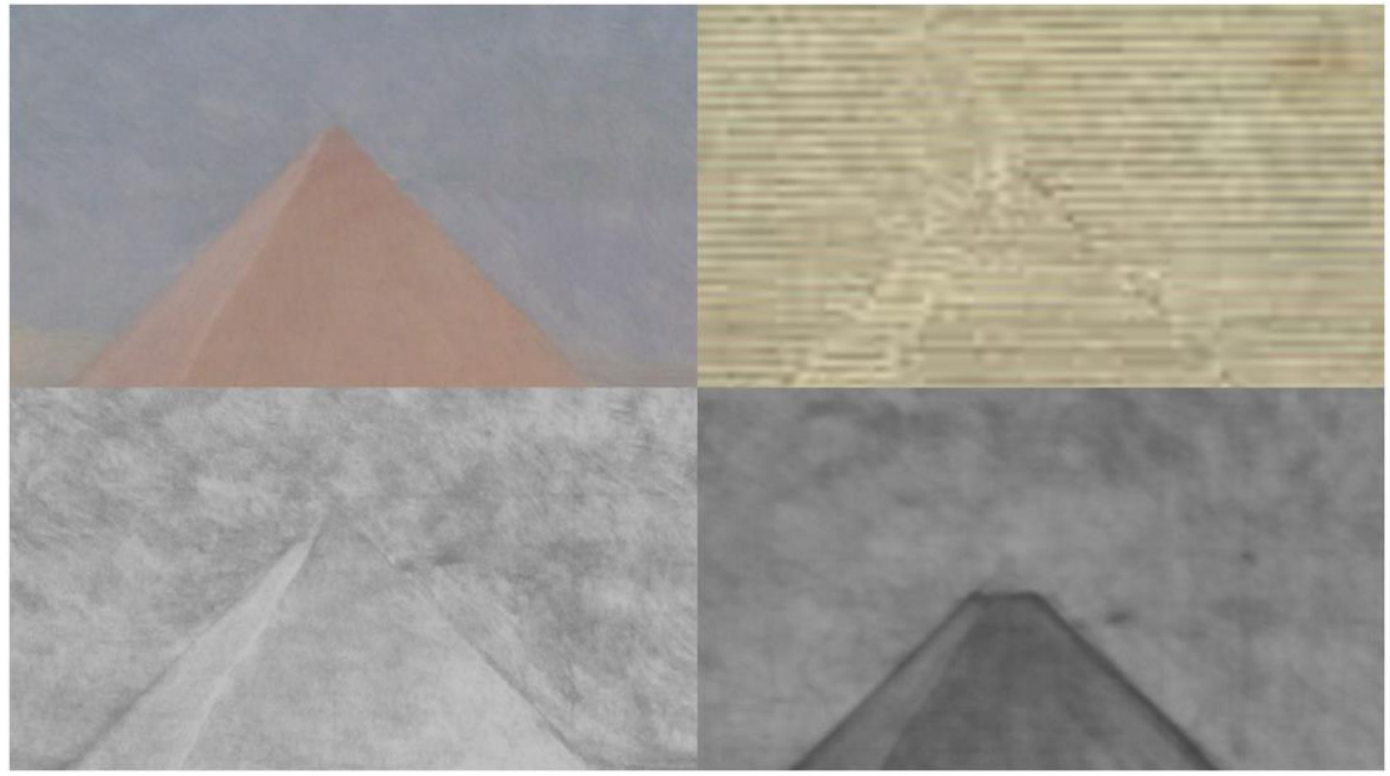

Figure 8 - Showing the tip of the pyramid in the interactive viewer. The two infrared images (bottom) show a line capping the top before the pinnacle. 
There is evidence that Poynter struggled to perfect some elements of the foreground in the drawing stage before painting. The rear of the red granite lion looks to have been elongated about three times with the plinth beneath it being reduced in length, shown in Figure 9. Similarly the curved tail of the whip held by the slave driver had numerous positions before he settled on a final outline which could be read more clearly and did not bisect the head of one of the processions attendants, shown in Figure 10.

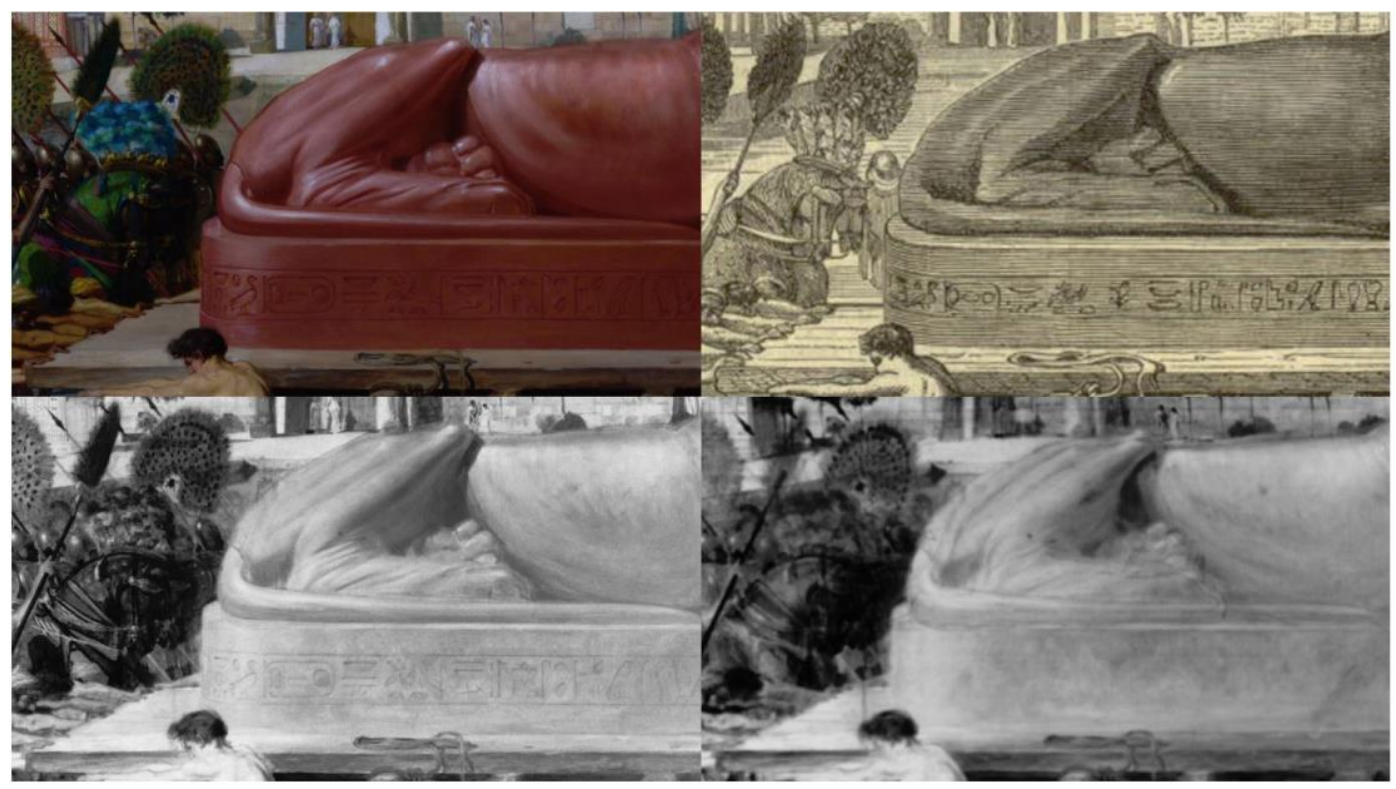

Figure 9 - Showing the rear of the lion in the interactive viewer. The infrared images (bottom) show changes made to the length of the lion and the plinth.

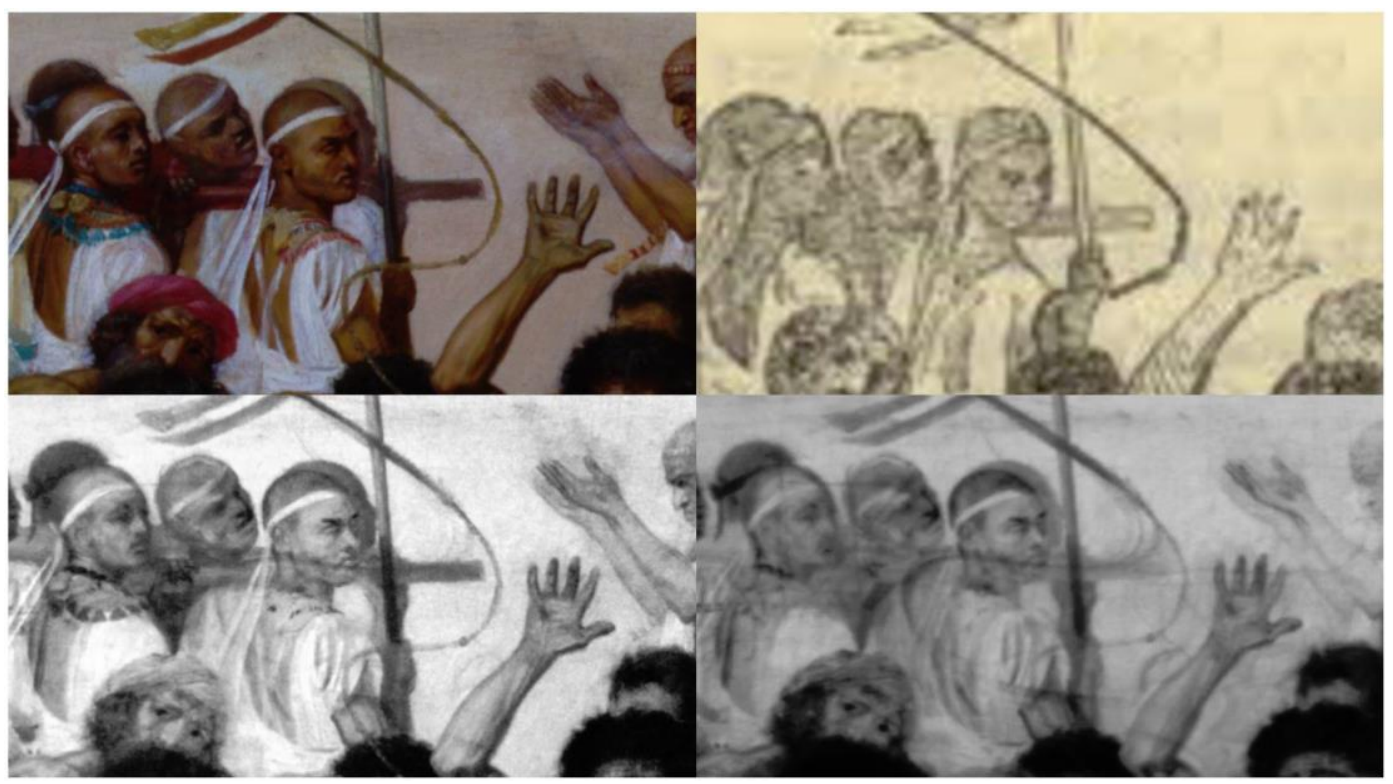

Figure 10 - Showing the whip in the interactive viewer. It is possible to see changes made to the position of the whip in the Osiris image (bottom right). 
The sky seems to have been painted using a combination of a highly reflective white ground, an IR absorbent pigment. The simple and relatively thin paint layer structure have made the artist's individual brush strokes clearly visible in 950 $\mathrm{nm}$ longpass images.

The sky has been built up of two applications, the first layer has been applied overall, the second application is only in certain areas intended to intensify the colour. The paint was applied in diagonal strokes with at least two stiff-haired brushes approximately 1 and $2 \mathrm{~cm}$ in width, the direction of which, from top left to bottom right, suggests the artist was righthanded. The strokes vary in length, in the first application the strokes are generally longer than those of the second application, where it appears the paint has been stippled on to the canvas, this can be clearly observed in the infrared 950 nm longpass images at the top edge of the canvas shown in Figure 11.

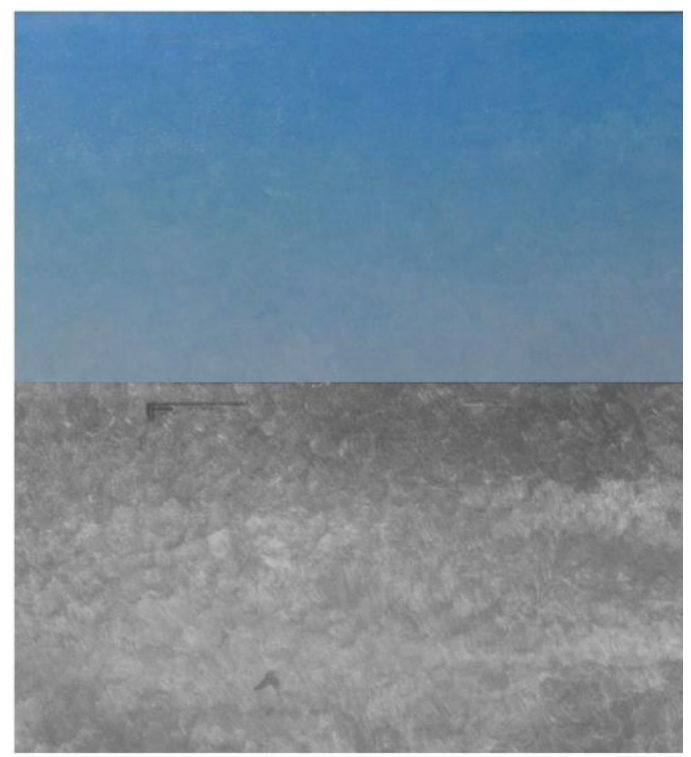

Figure 11 - Showing an area of the sky in the interactive viewer. It is possible to see the brushstrokes in the 950nm longpass image (bottom) and also an area of retouching.

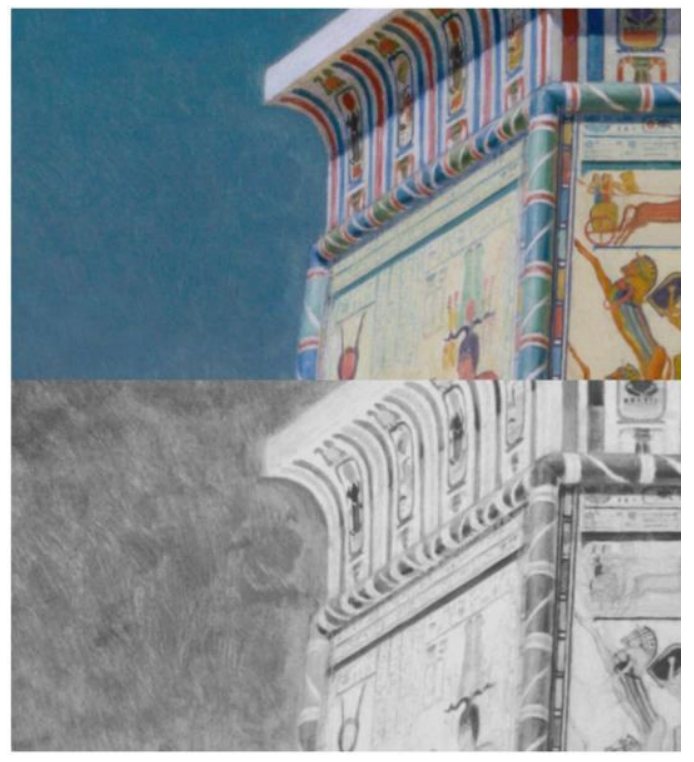

Figure 12 - Showing the sky against the edge of a building in the interactive viewer. In the $950 \mathrm{~nm}$ longpass image (bottom) it is possible to see the brush strokes used in the sky, and how these change towards the edge of the building.

The first application of blue paint can be seen to go neatly up to the edge of one of the buildings however the second application has been more loosely applied, falling short of the edge of the building in some areas but being used to sharpen the boundary of the sky and building in other areas. This is shown in Figure 12.

It is also possible to identify areas of modern retouching in instances where the pigment(s) used for the retouching differ in their reflectance in the infrared images. For example there are two small areas in the top left corner of the sky which have been retouched with a blue pigment which more strongly absorbs infrared and therefore appears darker than the original pigment in the $950 \mathrm{~nm}$ longpass infrared image, shown in Figure 11. 
Further analysis of the images leads to identification of ancillary figures which have been added to accompany the procession. These figures serve to increase the population density of the scene, filling the middle-ground and adding to the sense of recession in the scene. Comparison between the $950 \mathrm{~nm}$ longpass infrared and Osiris images reveals a subtle difference in the additional figures, which appear slightly fainter than the original figures in the Osiris image. The additional figure shown below in Figure 13 is a good illustration of this as all of the figures appear very similar in the visible image. This could be a result of different paint layers beneath the figures. The original figures are thought to be painted directly onto a light coloured priming on top of a simple grounding of chalk or gypsum in oil. The additional figures are painted over the priming and all of the subsequent paint layers. It is also possible to see original forms which have been covered with additional figures such as the second urn shown in Figure 14 that has been painted over another head.

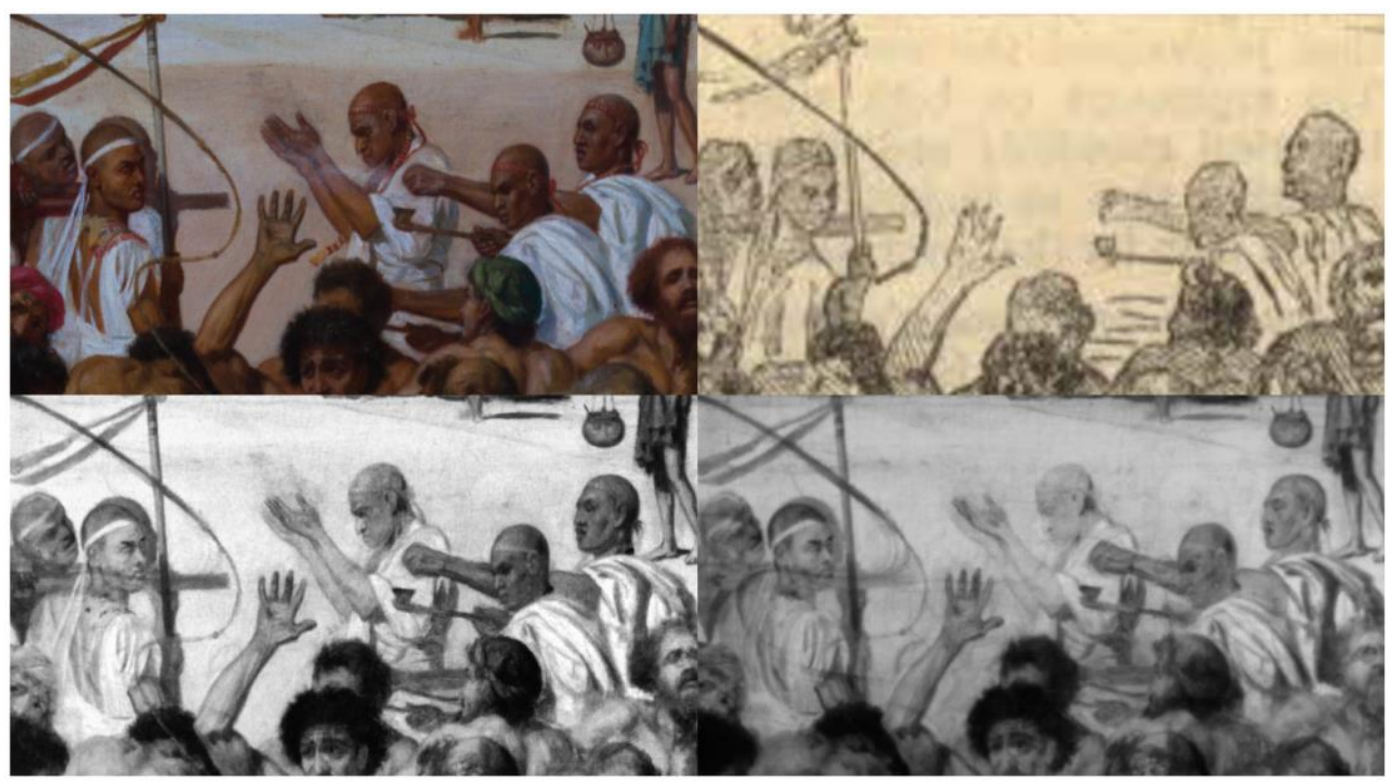

Figure 13 - Showing an additional figure in the interactive viewer.

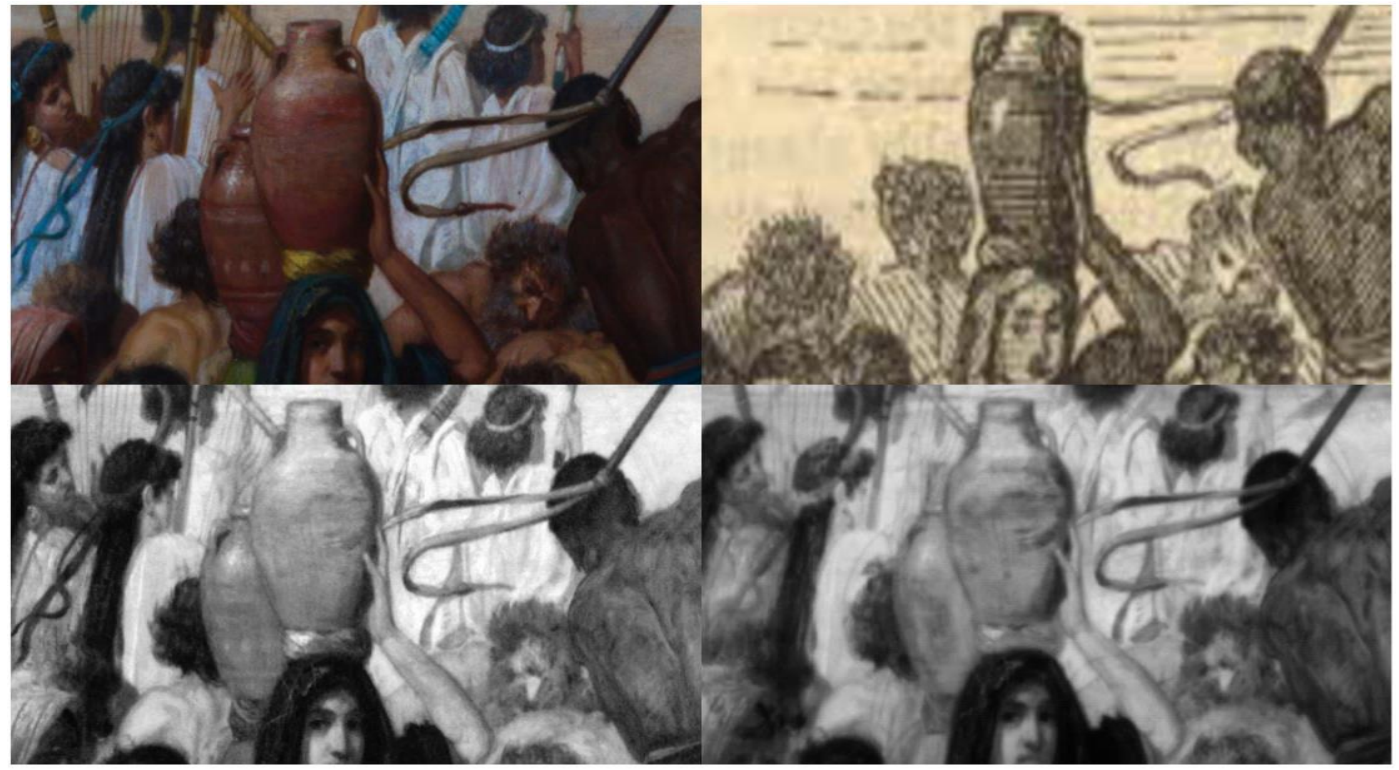

Figure 14 - Showing an urn painted over another figure in the interactive viewer. 


\section{CONCLUSIONS}

The visible, near-infrared, Osiris and Illustrated London News images have been registered together which enabled the images to be compared in an interactive viewer. The interactive viewer developed for this study has simplified comparison between the different image modalities. Comparisons between the $950 \mathrm{~nm}$ longpass infrared image and the Osiris infrared image proved useful in analysing the artist's technique across this painting. It has been possible to identify some composition changes using this tool to compare the visible and both infrared images with the woodcut engraving from the Illustrated London News. In particular, it was possible to identify changes and additions made after the initial underdrawing composition and initial paint layers such as changes to the position of the obelisk, the position of the whip, the pinnacle of the pyramid and additional figures in the scene.

This study shows that with relatively accessible and portable equipment such as a modified DSLR, the infrared $950 \mathrm{~nm}$ longpass images prove useful for analysing Poynter's technique for 'Israel in Egypt'. The Osiris images revealed more information about the composition and construction of the painting, and the infrared $950 \mathrm{~nm}$ image was useful for comparison. Future work could look to generate a higher resolution infrared image covering $900 \mathrm{~nm}-1700 \mathrm{~nm}$ at highresolution $(0.25 \mathrm{~mm} / \mathrm{pixel})$ over the whole painting.

\section{REFERENCES}

1. Israel in Egypt, Illustrated London News, London, 1868, pp. 84-85.

2. Israel in Egypt, Guildhall art gallery, city of london, accession number 1014.

3. The early history of the Illustrated London News, https://www.iln.org.uk/iln_years/earlyhistiln.htm

4. Illustrated London News, “ 2005,1129.21 ”www.Britishmuseum.org/collection , british museum, online. Accessed 28/09/2018

5. J. V. A. De Boer, Infrared reflectography: A method for the examination of paintings, Applied Optics 7 (1968), no. 9, 1711-1714.

6. C. M. Falco, High-resolution infrared imaging, SPIE Optics + Photonics2010.

7. A. Cosentino, Panoramic infrared reflectography. Technical recommendations, International journal of conservation science 5 (2014), no. 1, 51-60.

8. $\quad$ Opus Instruments, Osiris imaging system user manual, 2008.

9. Adobe digital negative converter, https://helpx.adobe.com/uk/photoshop/using/adobe-dngconverter.html

10. X-rite ColorChecker passport v1.1.1, https://www.xrite.com/servicesupport/downloads/c/colorchecker_camera_calibration_v1_1_1

11. RawTherapee v5.4, https://rawtherapee.com/

12. D. Mattes, D. R. Haynor, H. Vesselle, T. Lewellen and W. Eubank, Non-rigid multimodality image registration, Medical Imaging 2001: Image Processing, SPIE Publications, 2001, pp. pp. 1609-1620.

13. PTGui pro, https://www.ptgui.com/

14. H. Bay, T. Tuytelaars and L. Van Gool, Surf: Speeded up robust features, Computer visionECCV 2006 (2006), 404-417. 